\title{
Analyse des pertes rotoriques dissipées dans les machines synchrones associées à des convertisseurs statiques
}

\author{
D. Lechat, J. F. Rialland et R. Bonnefille
}

Conservatoire National des Arts et Métiers, Laboratoire d'Electricité Industrielle, 292, rue Saint Martin, 75141 Paris Cedex 03, France

(Reçu le 7 novembre 1983, révisé le 19 janvier 1984, accepté le 30 janvier 1984)

\begin{abstract}
Résumé. - Les pertes rotoriques dissipées dans une machine synchrone à pôles lisses et rotor massif sont déduites des courbes d'échauffement. L'analyse des pertes est effectuée en diversifiant les charges de la machine, fonctionnant en alternateur.

Les essais permettent de séparer les pertes liées aux harmoniques de dénture et celles qui sont associées au champ de réaction d'induit. On note que la saturation entraîne une accentuation des premières et un affaiblissement des secondes. Des relevés magnétiques confirment ces observations. L'analyse montre également l'importance de la distribution spatiale du champ de réaction d'induit sur le niveau des pertes.

Enfin, quelques mesures effectuées sur la machine munie d'un amortisseur mettent en évidence l'influence de ce dernier sur l'affaiblissement des champs harmoniques.
\end{abstract}

\begin{abstract}
The rotor losses in a synchronous machine with a slotted solid rotor were derived from transient temperature rise curves. The losses induced by the armature teeth and the additional losses caused by harmonic fields associated with the space non sinusoidal distribution and the harmonic components of phase currents were deduced from the values with different loads.

The saturation of the rotor involved an increase of the teeth harmonic losses and a decrease of the additional losses. The magnetic field harmonic components measured in the air-gap agree with the losses analysis. In this work, the influence of the field distribution in space is particularly discussed.

Some measurements were carried out with damper windings. The results show its influence on magnetic field and rotor losses.
\end{abstract}

\section{Introduction.}

La connaissance des pertes d'origine magnétique dans les machines électriques est un problème primordial puisqu'elle contribue, en effet, à la conception de machines de dimensionnement optimal. L'association de machines et de convertisseurs statiques, qui tend à se développer dans de nombreux domaines, rend le problème encore plus critique.

Dans le cas des machines synchrones à rotor massif, qui font l'objet de la présente étude, l'utilisation de l'électronique de puissance a pour conséquence la création d'harmoniques de courant et/ou de tension déformant le champ de réaction d'induit et entraînant des pertes électromagnétiques additionnelles, essentiellement par courants de Foucault, au rotor [1] et donc des échauffements supérieurs à ceux obtenus dans le cas d'une alimentation sinusoïdale.

Pour déterminer et analyser ces pertes, la méthode la plus prometteuse est celle qui fait appel à la modélisation magnétique de la machine, tout au moins lorsque les pertes par hystérésis et courants de Foucault peuvent être calculées séparément. Il est néanmoins important de pouvoir les évaluer par une autre méthode, basée en particulier sur des relevés expérimentaux. La mesure des températures permet, à l'aide d'un modèle thermique, de préciser la répartition des pertes au sein de la machine. Il faut noter que l'utilisation d'un modèle, nécessitant des hypothèses simplificatrices, limite la précision des résultats et implique la mesure de paramètres complémentaires (magnétiques par exemple).

Dans cet article, les pertes rotoriques, dissipées dans une machine synchrone à pôles lisses, sont déterminées à partir des courbes d'échauffement. L'analyse de ces pertes est rendue possible en diversifiant les charges de la machine, fonctionnant en alternateur. Nous avons tenté de mettre en évidence les 
avantages, les difficultés et les limites de cette approche, la première difficulté étant liée au fait que les pertes n'obéissent pas à des lois additives vis-à-vis des perturbations.

\section{Description du dispositif expérimental.}

2. 1 CARACTÉRISTIQUES DE LA MACHINE SYNCHRONE. La machine synchrone étudiée est tétrapolaire, triphasée, à rotor massif et d'une puissance nominale de $2,4 \mathrm{~kW}$.

Le stator, feuilleté, possède 48 encoches semifermées $(0,817)$, avec 8 conducteurs par encoche; l'enroulement est du type concentrique à pôles conséquents, à 4 encoches par pôle et par phase. Le diamètre intérieur est de $179 \mathrm{~mm}$, la longueur axiale de la partie active du fer de $78 \mathrm{~mm}$ et le pas polaire de $140,5 \mathrm{~mm}$. Le courant maximal admissible dans une phase est $J=13 \mathrm{~A}$. La résistance, mesurée à chaud, d'une phase statorique est $R_{\mathrm{s}}=0,218 \Omega$.

Le rotor, en acier doux, est massif et à pôles lisses. L'enroulement, du type imbriqué, est situé dans 24 encoches ouvertes, comportant chacune 35 conducteurs et réparties sur les $2 / 3$ du périmètre du rotor. Le maintien des conducteurs dans les encoches est assuré par des cales en bois pour les essais sans amortisseur, par des cales en laiton de $2,5 \mathrm{~mm}$ d'épaisseur lors des essais avec amortisseur (cales faisant alors partie de l'amortisseur lui-même). Le rotor a une longueur axiale de $86 \mathrm{~mm}$ et un diamètre de $178,2 \mathrm{~mm}$. Le courant maximal dans l'inducteur est $I_{\mathrm{e}}=10 \mathrm{~A}$. La résistance, mesurée à chaud, est $R_{\mathrm{e}}=1,8 \Omega$, valeur qui prend en compte la résistance de l'ensemble balais-bagues.

2.2 Montage Électrique DU BanC D'essai. - Le banc d'essai comprend l'alternateur précédemment décrit, le groupe qui entraîne la machine à la vitesse nominale de $1500 \mathrm{tr} . \mathrm{min}^{-1}$, les circuits de mesure et de charge. Les circuits de mesure sont classiques et ne seront donc pas décrits. L'alternateur a été soumis à 4 types d'essais : à vide, sur charge résistive, sur charge à prépondérance inductive, sur pont de Graetz commandé à thyristors. Dans ce dernier cas, le pont débite sur des rhéostats montés en série avec une inductance de lissage suffisante pour obtenir dans la charge un courant pratiquement continu. Des essais comparatifs ont, par ailleurs, été effectués avec et sans amortisseur afin de préciser son influence sur le niveau des pertes rotoriques.

2.3 CAPTEURS DE MESURES THERMIQUES ET MAGNÉTIQUES. - Les températures rotoriques sont mesurées à l'aide de 8 thermocouples 《chromel-alumel »; leur implantation est indiquée sur la figure 1. Les thermocouples sont reliés à un convertisseur tensionfréquence, mécaniquement solidaire du rotor. Les signaux issus du convertisseur sont transmis à l'aide d'un jeu de balais et de bagues. Ce dispositif, décrit en

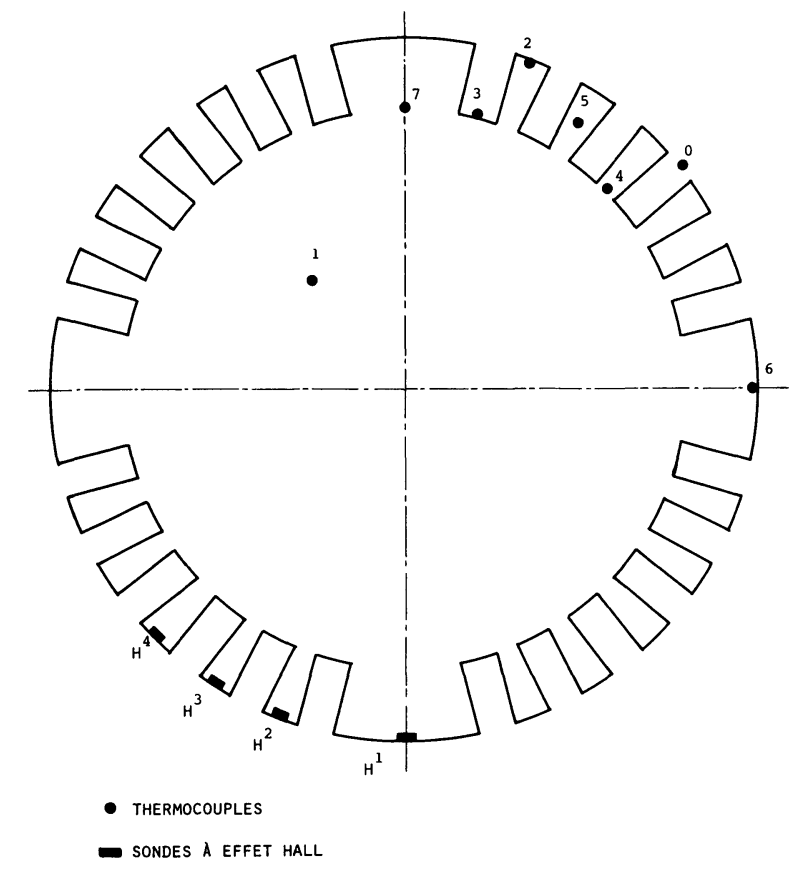

Fig. 1. - Vue frontale du rotor et implantation des capteurs.

[Cross-section of the rotor and location of thermocouples and Hall probes.]

détail dans un article précédent [2], permet la mesure d'échauffements jusqu'à $100 \mathrm{~K}$, avec une erreur inférieure à $1,5 \mathrm{~K}$.

Le stator est équipé de 3 thermocouples; deux sont implantés sur une flasque de l'induit et permettent d'apprécier la température de l'air issu de l'entrefer; le troisième, noyé au milieu d'une dent, donne la température de la surface rotorique. Les trois capteurs sont raccordés à une centrale de mesure, équipée d'un système de compensation de soudure froide.

La composante radiale de l'induction magnétique dans l'entrefer est mesurée par 4 sondes à effet Hall installées à la surface du rotor. Les sondes sont fixées dans des logements fraisés sur l'un des pôles et sur trois dents adjacentes (Fig. 1). La nécessité de pratiquer des rainures pour implanter les sondes a pour conséquence d'interdire la mesure des valeurs absolues de l'induction; seules les variations avec la position et/ou la charge seront significatives.

Le stator est équipé d'une bobine de mesure à pas polaire, constituée de 5 spires. Il est possible, à partir de la f.é.m. induite dans cette bobine, de déduire, avec certaines approximations, la répartition du champ synchrone à la surface du rotor.

\section{Modélisation thermique de la machine.}

Des essais préliminaires ont montré que les gradients thermiques au sein du rotor sont faibles et que celui-ci peut être considéré comme un solide homogène, de température moyenne $T_{\mathrm{r}}[2,3]$. 
L'analyse thermique du système nécessite de prendre en compte les échanges convectifs entre la surface latérale du rotor et l'entrefer $\left(T_{\mathrm{e}}\right)$ et les surfaces frontales et l'air ambiant $\left(T_{\mathrm{a}}\right)$. Ainsi, les pertes rotoriques et les différentes températures sont reliées par :

$$
p_{\mathrm{r}}=C \frac{\mathrm{d} T_{\mathrm{r}}}{\mathrm{d} t}+\alpha_{1}\left(T_{\mathrm{r}}-T_{\mathrm{e}}\right)+\alpha_{2}\left(T_{\mathrm{r}}-T_{\mathrm{a}}\right)
$$

où $C$ représente la capacité thermique du rotor, $\alpha_{1}$ et $\alpha_{2}$ les coefficients d'échange associés aux surfaces considérées.

La température de l'entrefer dépend également des échanges convectifs avec la surface du stator $\left(T_{\mathrm{s}}\right)$. En éliminant $T_{\mathrm{e}}$ de l'équation(1), on obtient en définitive :

$$
p_{\mathrm{r}}=C \frac{\mathrm{d} T_{\mathrm{r}}}{\mathrm{d} t}+\alpha\left(T_{\mathrm{r}}-T_{\mathrm{a}}\right)+K\left(T_{\mathrm{r}}-T_{\mathrm{s}}\right) .
$$

Dans cette équation, $\alpha$ apparaît comme un coefficient d'échange moyen et $K$ comme un coefficient correctif qui tient compte du couplage thermique du rotor et du stator par l'intermédiaire de l'entrefer. Lors de la détermination de $p_{\mathrm{r}}$, ces deux coefficients seront considérés comme des paramètres ajustables.

Pour chaque essai, les températures $T_{\mathrm{r}}, T_{\mathrm{s}}$ et $T_{\mathrm{a}}$ sont relevées au cours du temps. L'analyse de ces courbes permet de déduire, dans chaque cas, la valeur des pertes totales dissipées au rotor. Le troisième terme qui apparaît dans l'équation (2) étant toujours petit devant le second, l'échauffement rotorique est lié, avec une bonne approximation, à l'échauffement final $\left(T_{\mathrm{r}}-T_{\mathrm{a}}\right)_{\mathrm{f}}$ par une loi de la forme

$$
T_{\mathrm{r}}-T_{\mathrm{a}}=\left(T_{\mathrm{r}}-T_{\mathrm{a}}\right)_{\mathrm{f}}\left(1-\mathrm{e}^{-t / \tau}\right) .
$$

Plus précisément, les écarts entre les points expérimentaux et la meilleure courbe, calculée par la méthode des moindres carrés, sont toujours inférieurs aux erreurs de mesure. A titre d'exemple, les résultats correspondant à des essais à vide et sur pont de Graetz $\left(\psi=30^{\circ}\right)$ sont donnés sur la figure 2 .

Le calcul de la constante de temps $\tau$ permet, connaissant la valeur de $C$, d'en déduire $\alpha=C / \tau$. La détermination des pertes nécessite de connaître également la valeur de $K$. Celle-ci est obtenue de façon approchée à partir de deux essais, le premier à vide, rotor arrêté, le second à vide, rotor entraîné à $1500 \mathrm{tr}$. $\mathrm{min}^{-1}$. Dans le premier cas, les pertes rotoriques sont calculables; on trouve alors que $K$ vaut environ $1,4 \mathrm{~W} \cdot \mathrm{K}^{-1}$. Si l'on admet que $K$ varie avec la vitesse de rotation de la même manière que $\alpha$, on obtient $K=3 \mathrm{~W} \cdot \mathrm{K}^{-1}$ à $1500 \mathrm{tr} \cdot \mathrm{min}^{-1}$. Dans le second essai, $K$ est déterminé en supposant que les coefficients d'échange convectif entre le rotor et l'entrefer et entre le stator et l'entrefer sont du même ordre de grandeur. Un calcul élémentaire montre alors que [4] :

$$
\frac{K}{\alpha}=\frac{T_{\mathrm{e}}-T_{\mathrm{a}}}{T_{\mathrm{r}}+T_{\mathrm{s}}-2 T_{\mathrm{e}}} .
$$

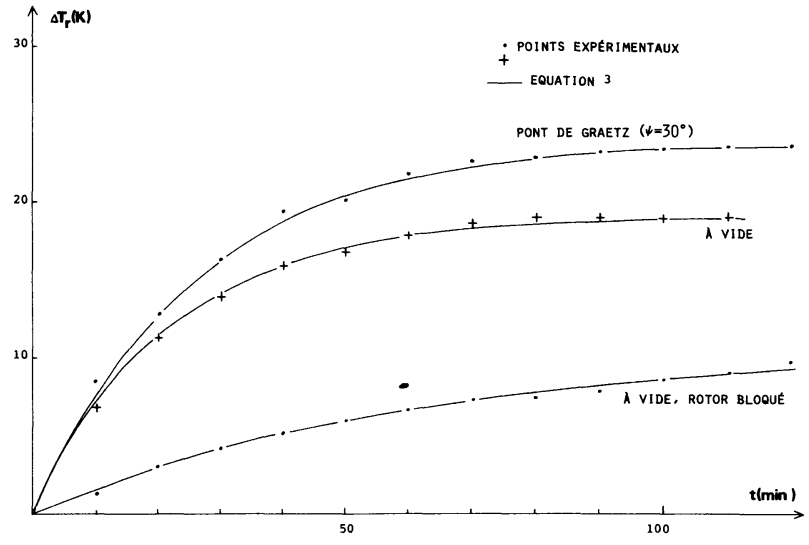

Fig. 2. - Echauffements rotoriques.

[Temperature-rises of the rotor.]

Les valeurs de $T_{\mathrm{e}}, T_{\mathrm{a}}, T_{\mathrm{r}}$ et $T_{\mathrm{s}}$ relevées à l'équilibre permettent, compte tenu de la valeur de $\alpha\left(7,5 \mathrm{~W} . \mathrm{K}^{-1}\right)$, d'obtenir l'ordre de grandeur de $K\left(2,9 \mathrm{~W} . \mathrm{K}^{-1}\right)$. Les valeurs ainsi déterminées ne sont qu'approximatives. Cependant, le fait que les deux calculs effectués selon des méthodes distinctes conduisent à des valeurs analogues justifie les approximations faites dans chaque cas. Par la suite, la valeur utilisée pour la détermination des pertes est $K=3 \mathrm{~W} . \mathrm{K}^{-1}$.

Lors de chaque essai, les pertes rotoriques sont calculées d'après l'équation :

$$
p_{\mathrm{r}}=C / \tau\left(T_{\mathrm{r}}-T_{\mathrm{a}}\right)_{\mathrm{f}}+K\left(T_{\mathrm{r}}-T_{\mathrm{s}}\right)_{\mathrm{f}} .
$$

Selon les essais, le dernier terme peut être positif ou négatif. Sa valeur absolue est toujours inférieure à $10 \%$ de $p_{\mathrm{r}}$. Cette contribution, relativement faible, s'explique par le fait qu'une part importante des pertes est évacuée par les parties frontales du rotor, zones où . l'influence du stator est négligeable et justifie, a posteriori, l'hypothèse formulee pour l'établissement de la relation (3). Les pertes, ainsi déterminées, sont obtenues avec une précision de 10 à $20 \%$.

\section{Détermination des pertes rotoriques.}

On peut distinguer 3 types de pertes dissipées au rotor : les pertes mécaniques $p_{\mathrm{m}}$, par effet Joule dans le bobinage inducteur $p_{\mathrm{J}}$, les pertes électromagnétiques $p_{\mathrm{e}}$ liées aux harmoniques de denture $\left(p_{\mathrm{h}}\right)$ et aux harmoniques du champ de réaction d'induit $\left(p_{\mathrm{s}}\right)$. Le but de l'analyse thermique est de séparer ces pertes et de préciser leur évolution selon les conditions de fonctionnement.

Un essai à $1500 \mathrm{tr} \cdot \mathrm{min}^{-1}$, inducteur non alimenté, permet de déterminer la fraction des pertes mécaniques qui contribue à échauffer le rotor. On trouve $p_{\mathrm{m}}=38 \mathrm{~W}$, alors que les pertes mécaniques totales sont de $77 \mathrm{~W}$.

Les pertes $p_{\mathrm{J}}$ sont calculables. Les écarts de tempé- 
rature selon les essais sont suffisamment faibles pour que les variations correspondantes de la résistance puissent être négligées.

4.1 Pertes par harmoniQues de Denture. - Les essais à vide permettent par différence entre $p_{\mathrm{r}}$ et $\left(p_{\mathrm{m}}+p_{\mathrm{J}}\right)$ d'obtenir les pertes par harmoniques de denture. La variation de $p_{\mathrm{h}}$ avec la valeur efficace $E$ de la f.é.m. induite dans une phase du stator est représentée sur la figure 3. La courbe $E\left(I_{\mathrm{e}}\right)$, non représentée, indique que la saturation apparaît pour $E>40 \mathrm{~V}$.

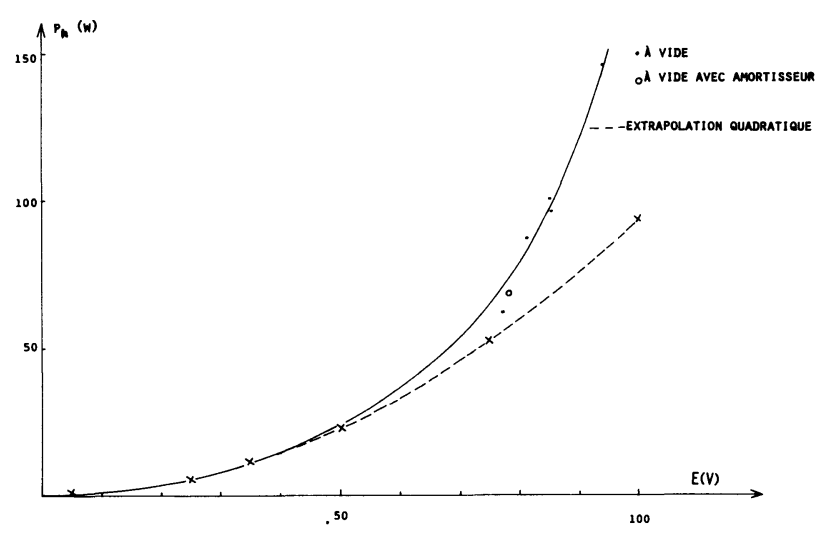

Fig. 3. - Pertes par harmoniques de denture.

[Harmonic losses induced by the armature teeth.]

En première approximation, les pertes par harmoniques de denture sont liées à $E, I_{\mathrm{e}}$ et $\Delta \mathcal{R}$, variation de la réluctance associée aux encoches statoriques, par une relation de la forme :

$$
p_{\mathrm{h}}=K \frac{E^{4}}{I_{\mathrm{e}}^{2}}(\Delta \mathcal{R})^{2} .
$$

Tant que la machine n'est pas saturée, $p_{\mathrm{h}}$ est proportionnel à $E^{2}$. La saturation entraîne, en général, une variation moins rapide de $p_{\mathrm{h}}$ avec $E$; lorsque les dents statoriques sont fortement saturées, on peut même observer une diminution de $p_{\mathrm{h}}$. Les résultats obtenus semblent contradictoires avec la remarque précédente. En fait, la variation traduit alors un comportement caractéristique des machines à encoches semi-fermées. La saturation, affectant en premier lieu les épaulements des dents, entraîne l'ouverture apparente des encoches et, par suite, s'accompagne d'une augmentation de $\Delta \mathcal{R}$. Pour illustrer ce phénomène, nous avons représenté sur la figure 4, la variation de $\Delta \mathcal{R}$, déduite de la relation (5), en fonction de $E$. Pour des valeurs de $E$ supérieures à celles mesurées, $\Delta \mathcal{R}$ doit passer par un maximum puis décroître lorsque la saturation des dents devient plus prononcée.

Les essais sans et avec amortisseur montrent que ce dernier n'a pas d'influence sur les pertes par harmoniques de denture (Fig. 3). L'analyse harmonique des signaux délivrés par les sondes à effet Hall montre que l'amplitude de l'harmonique $24(1200 \mathrm{~Hz})$, induit au

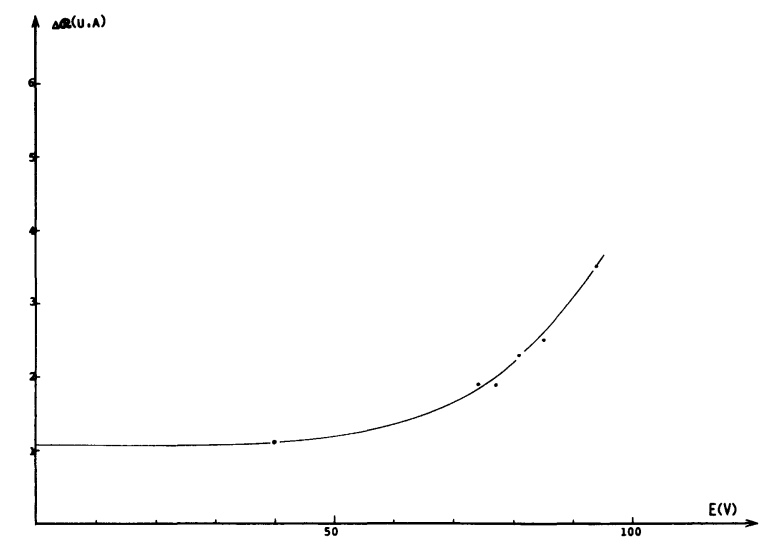

Fig. 4. - Influence de la f.é.m. à vide sur la variation de réluctance.

[Variation of reluctance versus no-load e.m.f.]

rotor par la denture statorique, n'est effectivement pas affaiblie par l'amortisseur. Cela provient du pas non adapté de l'amortisseur utilisé [5].

4.2 Pertes liées aux harmoniques du Champ De RÉACTION D'INDUIT. - En charge, il apparaît des pertes supplémentaires dues aux harmoniques de champ de réaction d'induit. Ces harmoniques peuvent avoir une double origine : distribution spatiale non sinusoiddale du champ dans l'entrefer (fonction de la répartition des bobinages statoriques et de la saturation des matériaux), existence d'harmoniques de courant. La combinaison des deux effets entraîne une répartition spatio-temporelle du champ de réaction d'induit particulièrement complexe. Ainsi, en négligeant la saturation des matériaux et les variations de réluctance et en se limitant aux premiers harmoniques (de rang 5), il apparaît un champ harmonique qui, dans un référentiel lié au rotor, peut se mettre sous la forme [4] :

$$
h(t, \beta)=\frac{3}{2} \hat{J}_{1} \hat{A}_{1} H(\beta) \cos (6 \omega t+\varphi(\beta))
$$

où $\hat{J}_{1}$ est le fondamental du courant, $\hat{A}_{1}$ le fondamental de la série associée à la distribution spatiale des courants statoriques, $\beta$ l'angle entre un point courant du rotor et l'axe du champ synchrone de réaction d'induit. En supposant que les amplitudes des harmoniques de rang 5 sont le $1 / 5$ de celles des fondamentaux, les variations de $H(\beta)$ et $h(t, \beta)$ pour $t=0$ sont représentées sur la figure 5 . Nous avons également porté les variations correspondantes lorsque la distribution des courants est sinusoïdale. Ces courbes sont évidemment idéales et ne tiennent pas compte de nombreux facteurs (en particulier l'existence des dents rotoriques).

L'utilisation de différentes charges permet de faire varier l'amplitude des harmoniques de courant. Sur pont de Graetz triphasé, on observe que le taux d'harmoniques varie avec l'angle d'amorçage $\psi$. Cela provient de la variation du temps de commutation 

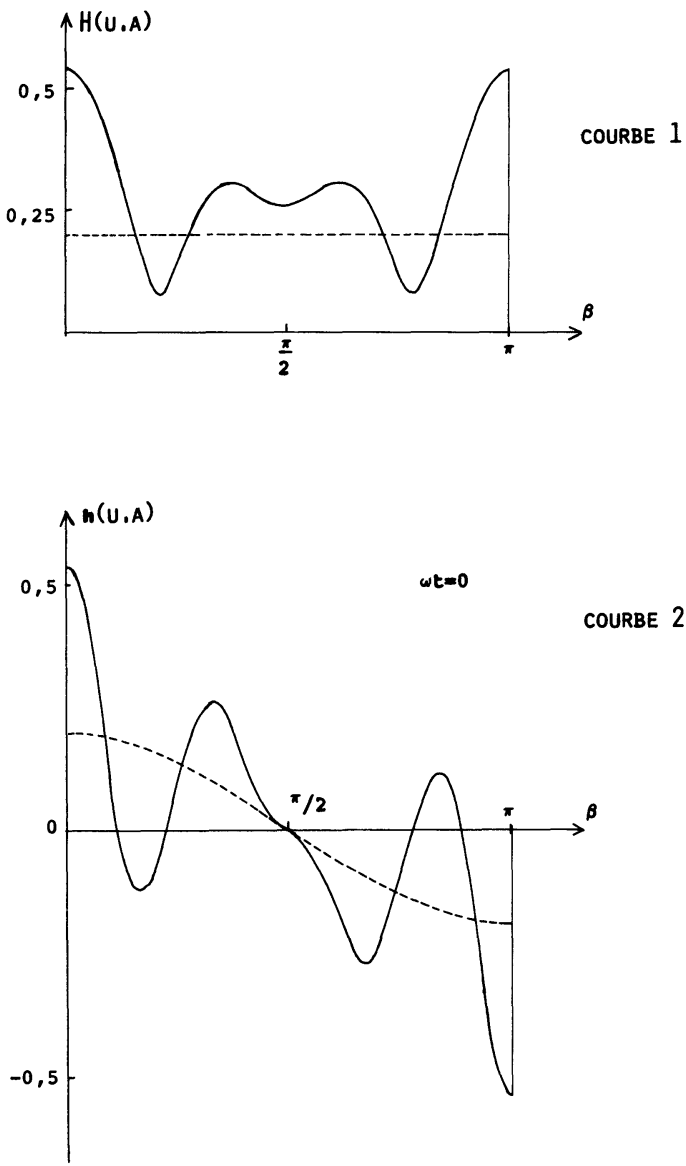

Fig. 5. - Champ harmonique de réaction d'induit dans un référentiel lié au rotor.

[Harmonic field distribution on the rotor surface.]

avec $\psi$ [6]. Nous avons représenté sur la figure 6 l'évolution des pertes électromagnétiques rotoriques $p_{\mathrm{e}}$ en fonction de l'amplitude de l'harmonique de rang 5; le courant d'excitation est de 4,4 A et la valeur efficace $J$ du courant statorique de $8 \mathrm{~A}$. Ces pertes, égales à la différence entre $p_{\mathrm{r}}$ et $\left(p_{\mathrm{m}}+p_{\mathrm{J}}\right)$, sont déduites des essais thermiques. Il est important de noter que la précision des mesures ne permet pas d'apprécier une évolution de la répartition des températures avec l'angle interne.

Nous avons tenté de séparer les pertes par harmoniques de denture et les pertes supplémentaires. Dans ce but, nous avons calculé, pour chaque essai, la valeur efficace $E_{\mathrm{r}}$ de la f.é.m. résultante. Nous avons supposé que la variation de $p_{\mathrm{h}}$ avec $E_{\mathrm{r}}$ est identique à celle donnée sur la figure 1 . Ainsi, les pertes liées à la réaction d'induit correspondent à la différence :

$$
p_{\mathrm{s}}=p_{\mathrm{r}}-\left(p_{\mathrm{m}}+p_{\mathrm{J}}\right)-p_{\mathrm{h}} \text {. }
$$

Deux remarques s'imposent. La première est que cette séparation peut apparaître comme artificielle, les deux effets n'étant pas nécessairement découplés. La deuxième est que la précision sur $p_{\mathrm{s}}$ est relativement faible; il ne faut considérer les résultats ainsi obtenus que comme des ordres de grandeur.
Nous avons porté, sur la figure 6, l'évolution de $p_{\mathrm{s}}$ avec $\hat{J}_{5}$. La dispersion des points ne permet pas de déduire une loi de variation précise. On constate que, sur charge $R-L$, ces pertes sont encore appréciables bien que les harmoniques de courant soient inexistants. Cela provient des harmoniques de distribution à l'origine de champs rampants [7]. Par ailleurs, on note que l'utilisation d'un amortisseur diminue sensiblement ces pertes, contrairement à ce qu'on observe en ce qui concerne les pertes par harmoniques de denture. De plus, l'essai sur pont de Graetz $\left(\psi=40^{\circ}\right)$ montre un effet sur $\hat{J}_{5}$ et donc sur les temps de commutation.

Nous avons représenté sur la figure 7 la variation de

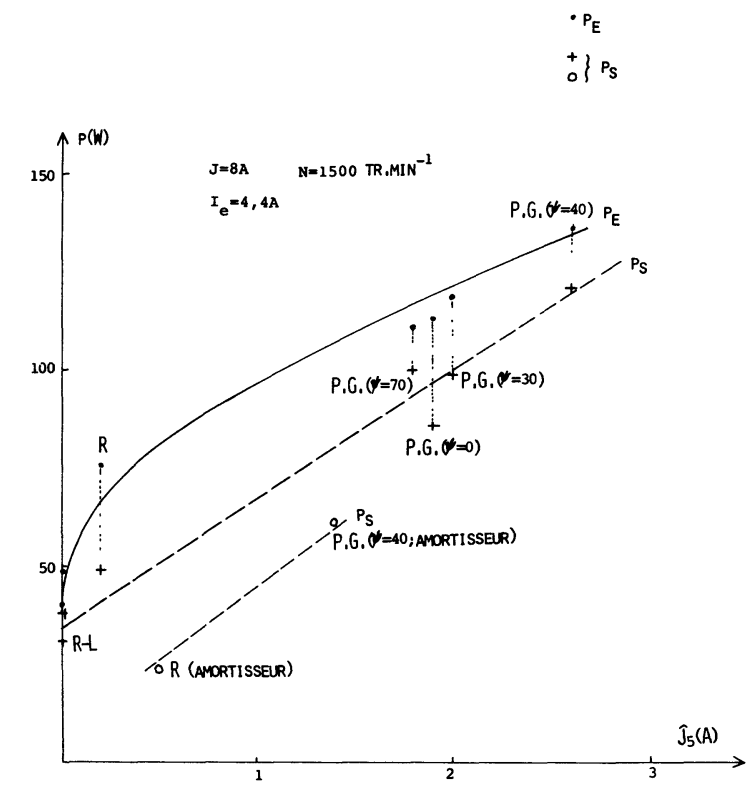

Fig. 6. - Pertes électromagnétiques et supplémentaires en fonction de l'amplitude de l'harmonique 5 de courant $\left(\bigcirc p_{\mathrm{e}} ;+\bigcirc p_{\mathrm{s}}\right)$.

[Electromagnetic and additional losses versus the 5th harmonic component of phase currents.]

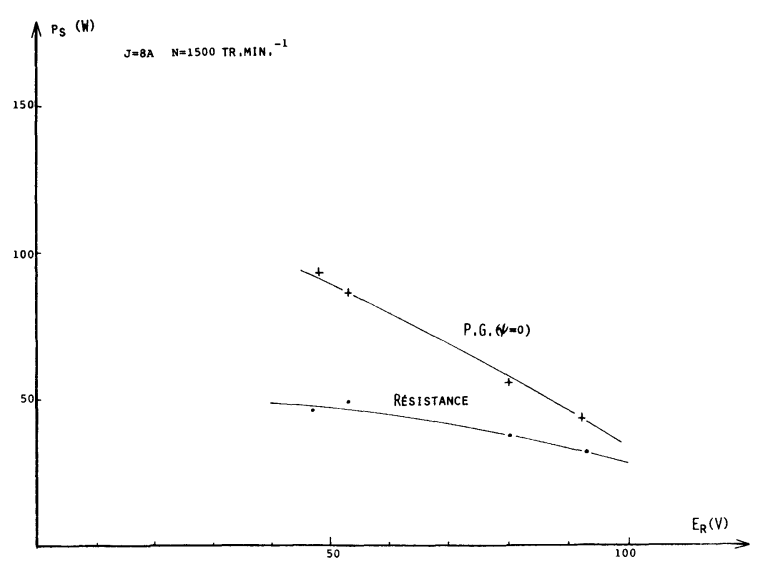

Fig. 7. - Pertes supplémentaires en fonction de la fé.m. résultante.

[Additional losses versus resultant e.m.f.] 
$p_{\mathrm{s}}$ avec la f.é.m. résultante pour deux charges. Les essais sont réalisés, pour $J=8 \mathrm{~A}$, en faisant varier le courant d'excitation. Sur charge résistive et sur pont de Graetz triphasé $(\psi=0)$, on observe une décroissance de $p_{\mathrm{s}}$ avec $E_{\mathrm{r}}$. Cela semble traduire une diminution de ces pertes lorsque la saturation du rotor augmente. Il faut cependant être prudent en ce qui concerne les résultats relatifs aux essais sur pont de Graetz, la variation de $E_{\mathrm{r}}$ s'accompagnant également d'une légère variation des temps de commutation. Cependant, un tel comportement s'explique dans la mesure où les courants de Foucault, responsables des pertes, sont directement liés à la perméabilité incrémentale du matériau [8] qui diminue avec la saturation.

\section{Analyse du champ dans l'entrefer.}

L'enregistrement de la tension $U_{\text {st }}(t)$, relevée à vide aux bornes de la bobine statorique à pas diamétral, est donné sur la figure 8. Lorsque le champ est fixe par rapport au rotor, cette courbe correspond à la répartition de la composante radiale de l'induction à la surface du rotor (aux fuites près). Ainsi, on peut observer la position relative des pôles, des dents et des encoches. Dans le cas présenté, l'induction dans l'axe polaire est d'environ $0,9 \mathrm{~T}$, la valeur moyenne sur un arc polaire de l'ordre de 0,6 T. En charge, l'existence de champs harmoniques ne permet pas de relier directement $U_{\text {st }}(t)$ et la répartition de l'induction à la surface du rotor. Néanmoins, l'examen des enregistrements fait apparaitre une déformation traduisant la variation de l'angle interne et l'inclinaison des lignes d'induction [4].

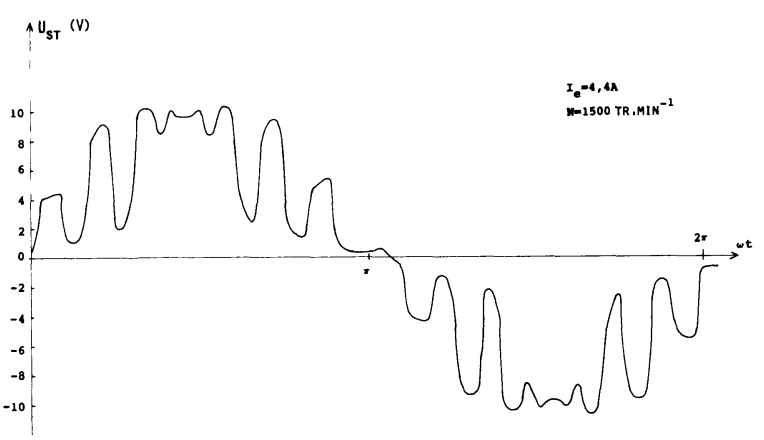

Fig. 8. - Enregistrement de la f.é.m. induite à vide dans une bobine statorique à pas diamétral.

[Recording of the no-load e.m.f. induced in a stator full pitch coil.]

L'analyse harmonique des signaux issus des sondes à effet Hall situées sur le rotor montre, à vide, l'existence d'un harmonique $24(1200 \mathrm{~Hz})$ de forte amplitude, lié à la denture statorique. En charge, apparaissent des harmoniques de rang moins élevé rendant compte des harmoniques du champ de réaction d'induit; parmi ceux-ci, celui de rang $6(300 \mathrm{~Hz})$ est le plus important.
Nous avons cherché à représenter les pertes, précédemment calculées, en fonction de l'amplitude de ces deux harmoniques $\left(V_{300}\right.$ et $\left.V_{1200}\right)$. La difficulté vient de ce que, selon les essais, de nombreux facteurs influent sur les signaux. En ce qui concerne l'harmonique 24, il apparaît que l'inclinaison des lignes d'induction avec la charge entraîne une saturation locale des dents qui ne permet pas de corréler l'amplitude de cet harmonique avec la " saturation moyenne " du rotor. C'est pourquoi nous avons choisi de caractériser l'harmonique 24 à partir des signaux issus de la sonde située sur l'axe polaire (sonde no 1 , Fig. 1). Nous avons porté, sur la figure 9 , les valeurs des pertes dues

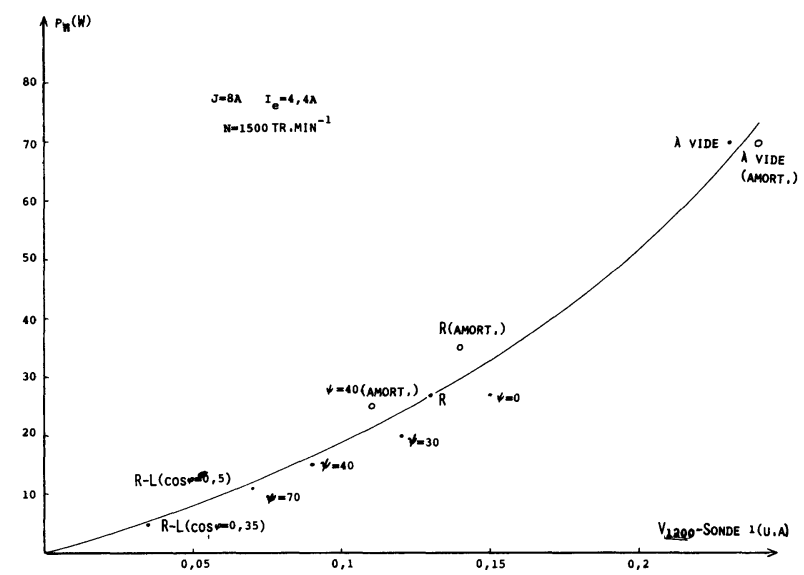

Fig. 9. - Pertes par harmoniques de denture en fonction de l'amplitude de l'harmonique 24 induit au rotor.

[Teeth harmonic losses versus the 24th harmonic component induced in the rotor.]

aux harmoniques de denture, calculées pour différents essais réalisés avec le même courant d'excitation $I_{\mathrm{e}}$ et le même courant d'induit $J$, en fonction de l'amplitude de l'harmonique 24, notée $V_{1200}$. Cette figure fait apparaître une corrélation entre $p_{\mathrm{h}}$ et $V_{1200}$ qui tend à confirmer les hypothèses formulées dans les 2 cas. Les pertes varient approximativement avec $V_{1200}$ selon une loi de la forme :

$$
p_{\mathrm{h}}=k\left(V_{1200}\right)^{1,5} .
$$

Il est difficile de discuter précisément de la valeur de l'exposant, dans la mesure où la répartition des lignes d'induction est notablement différente à vide et en charge et selon la charge. Enfin, les résultats de la figure 9 confirment le peu d'influence de l'amortisseur sur les phénomènes liés aux harmoniques de denture.

Dans le cas de l'harmonique 6, l'analyse des signaux est encore plus délicate. En effet, la figure 5 montre que, pour un essai donné, l'amplitude de cet harmonique dépend fortement de la position de la sonde sur le rotor. Encore faut-il rappeler que les courbes sont calculées avec des hypothèses simplificatrices. Lorsqu'on utilise différentes charges, l'angle entre l'axe du champ de réaction d'induit et l'axe polaire n'est pas 
constant. Ainsi, les variations de l'amplitude de l'harmonique 6 avec la charge peuvent traduire le glissement des courbes de la figure 5 par rapport aux sondes. La courbe 1 montre néanmoins une zone où $H$ varie peu avec $\beta$. Pour tous les essais effectués, seule la sonde $n^{0} 3$ (Fig. 1) est localisée dans cette zone. Sur la figure 10 sont portées les valeurs de $p_{\mathrm{s}}$ en fonction de l'amplitude de l'harmonique 6 détecté par cette sonde $\left(V_{300}\right)$. Contrairement au cas présenté, il n'est pas possible de corréler $p_{\mathrm{s}}$ et les amplitudes $V_{300}$ associées aux autres sondes. Il faut enfin préciser que des facteurs, tels que l'inclinaison des lignes d'induction et la saturation hétérogène des dents, affectent également les valeurs relevées. Malgré ces limitations, la figure 10 montre que, à une augmentation de

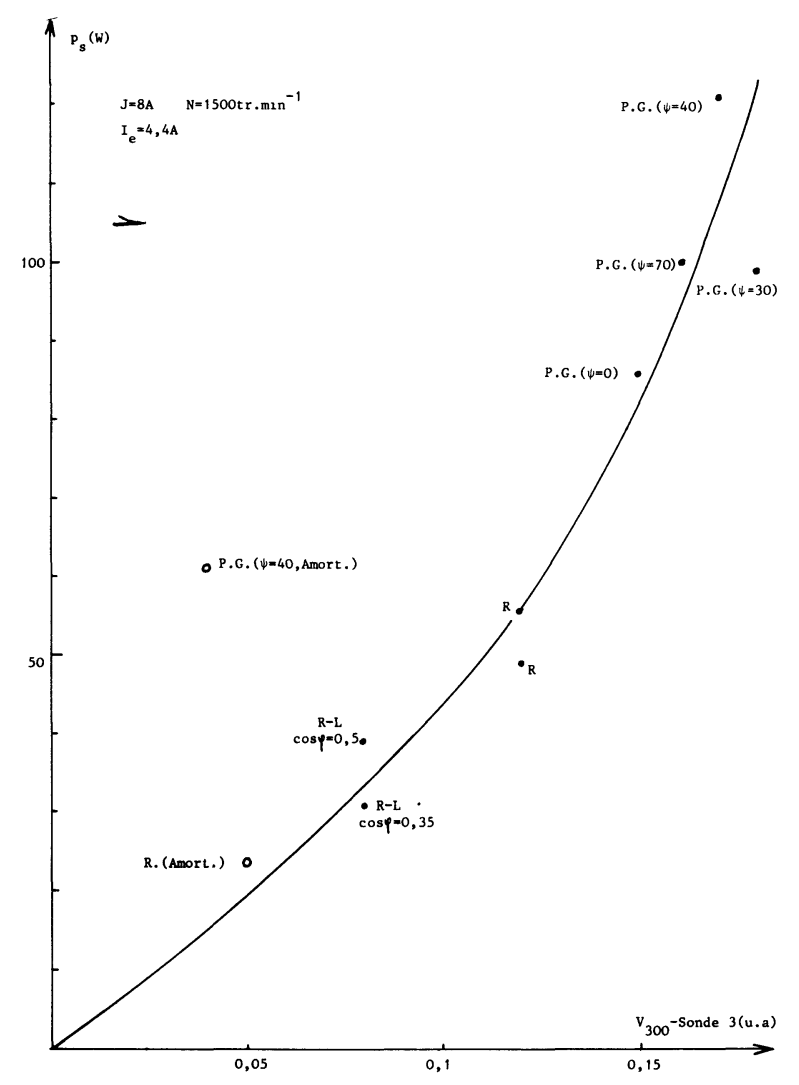

Fig. 10. - Pertes supplémentaires en fonction de l'amplitude de l'harmonique 6 induit au rotor.

[Additional losses versus the 6th harmonic induced in the rotor.]

$V_{300}$, associée à l'harmonique du champ de réaction d'induit le plus important, correspond effectivement un accroissement des pertes. On note que, sur charge $R-L$, les pertes dues aux seuls champs rampants sont encore importantes. Par ailleurs, la comparaison des essais sur pont de Graetz $(\psi=40)$ avec et sans amortisseur met en évidence un affaiblissement des harmoniques de champ et une diminution des pertes correspondantes. De plus, l'écart entre les pertes calculées selon (7), en présence de l'amortisseur, et la courbe moyenne, tracée sur la figure 10 , peut traduire l'existence des pertes par effet Joule dissipées dans les barres d'amortisseur.

Si l'analyse du champ dans l'entrefer ne permet pas d'obtenir de façon précise la répartition spatiotemporelle, elle conduit à des corrélations avec les pertes qui semblent confirmer les hypothèses nécessaires à leur séparation.

\section{Conclusion.}

L'étude réalisée montre que l'analyse thermique d'une machine permet d'évaluer et de localiser les pertes et, dans une certaine mesure, de les différencier selon leur origine. La précision des résultats est, en premier lieu, fonction du nombre de capteurs utilisés. Par ailleurs, le modèle thermique conduisant aux relations entre les pertes et les échauffements fait intervenir des échanges thermiques entre les différentes zones de la machine qu'il est parfois difficile de caractériser avec précision. Les pertes, déduites de tels essais, sont particulièrement sensibles à la valeur attribuée aux coefficients d'échange $[9,10]$. Cela constitue le point faible de cette approche. Il n'en demeure pas moins que les résultats obtenus donnent des renseignements complémentaires de ceux qu'on peut déduire d'essais électromécaniques. Ainsi, dans l'exemple traité, apparaît l'importance de la saturation sur les pertes par harmoniques de denture et celles associées au champ de réaction d'induit. De même, l'influence de l'amortisseur est mieux analysée. Les mesures du champ dans l'entrefer, bien que difficiles à interpréter, semblent confirmer les conclusions de l'étude thermique.

Afin de préciser les pertes dissipées dans des machines synchrones du fait de leur association avec des convertisseurs statiques, nous envisageons de réaliser une machine mieux adaptée que celle utilisée ici. En effet, l'analyse claire du problème nécessite de minimiser les effets liés à la denture et à la distribution non sinusoïdale du champ. De plus, l'augmentation des coefficients d'échange thermique (entre le rotor et le stator, entre les bobinages et le matériau magnétique, ...) permettra, en découplant les effets thermiques, d'obtenir une meilleure précision. Nous pensons qu'alors une comparaison significative pourra être effectuée entre les pertes déduites d'une telle étude et celles calculées à partir du champ électromagnétique, en particulier, dans le cas d'une machine équipée d'un amortisseur. 


\section{Bibliographie}

[1] Abdel-Razek, A., Additional losses in solid rotor synchronous machines with static convertors, E.M.E. 5 (1980) 101.

[2] Garcia, R., Mesiere, J., Rialland, J. F., BonneFILLE, R., Détermination des températures dans les parties tournantes des machines électriques. Application aux machines synchrones à rotor massif, R.G.E. 22, 1 (1982) 21.

[3] Horn, L., Rialland, J. F., Bonnefille, R., AbdelRAZEK, A., Heating and rotor losses in synchronous machines controlled by power electronic devices, Proc. of ICEM, Budapest, HC5 (1982) 361.

[4] Lechat, D., Contribution à la modélisation thermique des machines synchrones, Mémoire d'Ingénieur CNAM, Paris (1983).

[5] Guilbert, A., Machines synchrones (Dunod éd., Paris) 1965.
[6] Abdel-RazeK, A., Natural commutation in solid rotor synchronous machines associated with static convertors, E.M.E. 4 (1979) 331.

[7] Seguier, G. et Notelet, F., Electrotechnique industrielle (Technique et Documentation éd., Paris) 1977.

[8] Woods, E., Eddy current loss on solid iron with D. C. offset, IEEE PAS-100, 5 (1981) 2241.

[9] Bahbouth, S., Etude en régime permanent d'une machine asynchrone alimentée par un onduleur à transistors. Performances, pertes, échauffement. Thèse de Docteur Ingénieur, INP de Grenoble (1981).

[10] Namburi, N. R., Barton, T. H., Thermal modelling of an induction motor, IEEE PAS-102, 8 (1983) 2636. 